\title{
Biofilme em feridas: conhecimento do enfermeiro na avaliação e tratamento
}

\author{
Biofillm in wounds: nurses' knowledge in evaluation and treatment \\ Biofilm en heridas: conocimiento de enfermeras en evaluación y tratamiento
}

Recebido: 29/09/2021 | Revisado: 02/10/2021 | Aceito: 07/10/2021 | Publicado: 10/10/2021

\author{
Thamiris Leal Coimbra \\ ORCID: https://orcid.org/0000-0003-3896-8634 \\ Universidade Federal de Mato Grosso do Sul, Brasil \\ E-mail: tha.aique@gmail.com \\ Carolina Mariano Pompeo \\ ORCID: https://orcid.org/0000-0003-4454-0140 \\ Universidade Federal de Mato Grosso do Sul, Brasil \\ E-mail: carolmpompeo@gmail.com \\ Mercy da Costa Souza \\ ORCID: https://orcid.org/0000-0001-9323-599X \\ Universidade Federal de Mato Grosso do Sul, Brasil \\ E-mail: me.enfmercy@gmail.com \\ Ramon Moraes Penha \\ ORCID: https://orcid.org/0000-0002-8656-2535 \\ Universidade Federal de Mato Grosso do Sul, Brasil \\ E-mail: ramon.penha@ufms.br
}

\begin{abstract}
Resumo
Objetivo: Identificar o conhecimento de enfermeiros acerca do biofilme em feridas complexas. Método: Estudo descritivo e transversal, realizado em cinco unidades de internação de pacientes adultos em um hospital público de ensino no estado de Mato Grosso do Sul. Para a coleta de dados foi utilizado um instrumento semiestruturado, autoaplicável, composto por 26 questões. Este estudo foi aprovado pelo Comitê de Ética em Pesquisa em seres humanos da Universidade Federal de Mato Grosso do Sul sob parecer número 3.727.518. Resultados: Participaram da pesquisa 52 profissionais, com média de idade de 37,63 anos e predomínio do sexo feminino. A maioria possuía experiência na área e atuava na unidade de urgência e emergência, no turno diurno e tinha como maior grau de formação a especialização. Quanto ao recebimento de treinamento acerca do biofilme, a maioria relatou não ter recebido. As perguntas com maior percentual de respostas incorretas estavam relacionadas ao diagnóstico e tratamento. Conclusão: Os participantes apresentaram limitações no conhecimento acerca do biofilme em feridas complexas, o que pode influenciar na prática clínica. Acredita-se que a falta de atividades voltadas ao ensinoaprendizagem na instituição tenha contribuído para estes resultados.
\end{abstract}

Palavras-chave: Feridas; Biofilme; Enfermagem; Curativo.

\begin{abstract}
Objective: To identify nurses' knowledge about biofilm in complex wounds. Method: Descriptive and cross-sectional study, carried out in five inpatient units of adult patients in a public teaching hospital in the state of Mato Grosso do Sul. For data collection, a semi-structured, self-administered instrument consisting of 26 questions was used. This study was approved by the Human Research Ethics Committee of the Federal University of Mato Grosso do Sul under opinion number 3.727.518. Results: 52 professionals participated in the research, with a mean age of 37.63 years and a predominance of females. Most had experience in the area and worked in the urgency and emergency unit, in the day shift and had specialization as their highest level of training. As for receiving training about biofilm, most reported not having received it. The questions with the highest percentage of incorrect answers were related to diagnosis and treatment. Conclusion: Participants had limitations in knowledge about biofilm in complex wounds, which can influence clinical practice. It is believed that the lack of activities aimed at teaching and learning in the institution has contributed to these results.
\end{abstract}

Keywords: Wounds; Biofilm; Nursing; Band Aid.

\section{Resumen}

Objetivo: Identificar el conocimiento de las enfermeras sobre el biofilm en heridas complejas. Método: Estudio descriptivo y transversal, realizado en cinco unidades de internación de pacientes adultos en un hospital público docente del estado de Mato Grosso do Sul. Para la recolección de datos se utilizó un instrumento autoadministrado semiestructurado de 26 preguntas. usó. Este estudio fue aprobado por el Comité de Ética en Investigación en Humanos de la Universidad Federal de Mato Grosso do Sul con el número de opinión 3.727.518. Resultados: participaron de la investigación 52 profesionales, con una edad media de 37,63 años y predominio del sexo femenino. 
La mayoría tenía experiencia en el área y trabajaba en la unidad de urgencia y emergencia, en el turno de día y tenía la especialización como su nivel más alto de formación. En cuanto a recibir formación sobre biofilm, la mayoría informó no haberla recibido. Las preguntas con mayor porcentaje de respuestas incorrectas se relacionaron con el diagnóstico y el tratamiento. Conclusión: Los participantes tenían limitaciones en el conocimiento sobre el biofilm en heridas complejas, lo que puede influir en la práctica clínica. Se cree que la falta de actividades orientadas a la enseñanza y el aprendizaje en la institución ha contribuido a estos resultados.

Palabras clave: Heridas; Biopelícula; Enfermería; Apósito adhesivo.

\section{Introdução}

$\mathrm{O}$ termo feridas complexas se refere às feridas agudas e/ou crônicas que frequentemente desafiam os profissionais de saúde, devido ao grande comprometimento tecidual, a presença de infecção e a consequente formação de biofilme (Jara, Silva, Zanchetta, Roto, \& Lima, 2017).

Nesse contexto, o aumento na expectativa de vida da população associado à presença de doenças crônicas não transmissíveis (DCNT) têm contribuído para o desenvolvimento de feridas complexas. Estas, decorrem muitas vezes de fatores locais e/ou sistêmicos ao indivíduo que prejudicam o processo de reparação tecidual e contribuem para a estagnação das lesões, muitas vezes decorrentes da presença de biofilme (Duim, Sá, Duarte, Oliveira, \& Lebrão, 2015).

Os biofilmes são estruturas microscópicas, constituídos por um encapsulado de micro-organismos de diferentes espécies microbianas que interferem diretamente na resposta imunológica do indivíduo. Quando presentes na lesão, impedem o reconhecimento e a subsequente eliminação dos micro-organismos, o que contribui para o aumento do risco de tolerância e resistência ao tratamento proposto (Mihai, Preda, Lungu, Gestal, Popa, \& Holban, 2018).

Associam-se principalmente à dificuldade no processo de cicatrização que decorre da inflamação crônica local. É mais prevalente em feridas crônicas, no entanto pode ser encontrado em feridas agudas, incluindo as feridas operatórias (Ferro, et al., 2019).

$\mathrm{O}$ cuidado com feridas faz parte da rotina da equipe de enfermagem, em especial do enfermeiro, que possui competência técnica e científica para atuar nas ações de prevenção e tratamento (Franco, et al., 2018). Diante disso, torna-se necessário o aprimoramento constante destes profissionais, uma vez consideradas as diferentes modalidades terapêuticas e a infinidade de coberturas disponíveis no mercado (Agra, et al., 2017).

Entretanto, embora o enfermeiro seja um dos profissionais de maior destaque junto ao tratamento das lesões de pele, em especial às feridas complexas, o quantitativo de estudos que abordem o conhecimento destes profissionais frente ao diagnóstico e tratamento do biofilme no Brasil ainda é limitado e insuficiente para contribuir de forma efetiva na melhoria deste conhecimento.

Assim, esta pesquisa justifica-se pela escassez de estudos na literatura científica que abordem a temática e que possam efetivamente contribuir com a melhoria do cuidado às pessoas com feridas complexas, uma vez que o conhecimento e a prática deficitária exercida pelos enfermeiros podem refletir diretamente na qualidade da assistência prestada.

Por fim, este estudo foi desenvolvido a fim de responder à questão norteadora: O que os enfermeiros sabem acerca do biofilme em feridas complexas? Diante do exposto e da importância da temática na saúde pública, o objetivo deste estudo é identificar o conhecimento de enfermeiros acerca do biofilme em feridas complexas.

\section{Metodologia}

Trata-se de um estudo descritivo, analítico, de delineamento transversal e abordagem quantitativa, realizado em cinco unidades de internação de pacientes adultos de um hospital público de ensino em Campo Grande, Mato Grosso do Sul.

Foram incluídos na pesquisa todos os enfermeiros que atuassem diretamente nos setores de internação de clínica médica, enfermaria de doenças infecciosas e parasitárias, clínica cirúrgica 1, clínica cirúrgica 2 e pronto atendimento médico. 
Foram excluídos os profissionais que estivessem afastados do serviço por férias ou atestado médico e não foram incluídos os que eventualmente estivessem lotados nos setores em decorrência de remanejamentos ou plantões extras.

A amostra foi composta pela totalidade de enfermeiros lotados nos locais de estudo e que aceitaram participar da pesquisa, portanto não foi realizado cálculo amostral.

Os participantes foram identificados por meio das escalas de serviço das unidades elencadas. A coleta de dados ocorreu no período de janeiro a outubro de 2020, nos três turnos de trabalho, com entrevista em horários pactuados com os participantes de modo a não atrapalhar a rotina de serviço dos profissionais. Antes da entrevista e da inclusão no estudo, os participantes receberam informações sobre os objetivos da pesquisa e os que aceitaram participar assinaram o Termo de Consentimento Livre e Esclarecido (TCLE).

Para a coleta de dados, foi utilizado um instrumento semiestruturado, autoaplicável, elaborado pelas pesquisadoras com base na literatura científica (Percival, Vuotto, Donelli, \& Lipsky, 2015; Wei et al., 2019; Murphy et al., 2020) composto por 26 questões organizadas em três categorias.

A primeira aborda a caracterização sociodemográfica dos participantes enquanto a segunda, refere-se à caracterização profissional e contém questões desde a formação acadêmica até o tempo de experiência na área. A terceira e última parte do instrumento aborda o conhecimento do enfermeiro acerca do biofilme. É composta por sete itens organizados em questões discursivas e de múltipla escolha.

Para responder a última questão, os participantes receberam além do instrumento semiestruturado, quatro imagens de feridas complexas disponibilizadas no site Google imagens e impressas em papel fotográfico. Nesta questão, os participantes realizaram a avaliação clínica das lesões e assinalaram as opções que indicavam haver a presença de biofilme.

As respostas das questões discursivas foram analisadas e categorizadas em corretas ou parcialmente corretas e incorretas. Foram consideradas corretas as respostas que se encontravam integralmente de acordo com a literatura consultada; parcialmente correta, as respostas incompletas e/ou que não estavam em total conformidade com a literatura base, mas que não continham afirmativas contrárias à base científica; e incorretas, as respostas que estavam em total inconformidade com a literatura adotada. Quanto às questões de múltipla escolha, a pergunta número 24 possuía apenas uma resposta correta, enquanto as demais possuíam duas alternativas corretas.

Para garantir a consistência do instrumento, foi realizado um teste piloto com dez enfermeiros lotados em setores do referido hospital que não faziam parte do cenário da pesquisa. Primeiramente os profissionais foram orientados acerca da pesquisa, e os que puderam colaborar, responderam ao questionário de acordo com o seu entendimento. Após o teste piloto, foram realizados ajustes no instrumento de maneira a deixá-lo mais objetivo para os participantes da pesquisa.

Para a análise estatística, a comparação das respostas entre enfermeiros sobre o conhecimento acerca do biofilme em feridas complexas em relação à idade, ao tempo de experiência profissional e ao tempo de atuação desses no setor, foi realizada por meio do teste t-student.

A associação entre as variáveis sexo, turno de trabalho, recebimento de treinamento acerca do biofilme e a frequência com que os enfermeiros se deparavam com as feridas complexas foi analisada por meio do teste exato de Fisher e a avaliação da associação entre as variáveis pós-graduação e setor de atuação foi realizada por meio do teste do qui-quadrado.

Os resultados foram apresentados na forma de estatística descritiva e em tabelas. A análise estatística foi realizada utilizando-se o programa estatístico Statistical Package for the Social Sciences (SPSS), versão 24.0. Para todos os testes foi considerado um nível de significância de 5\%.

Esta pesquisa foi aprovada pelo Comitê de Ética em Pesquisa em seres humanos da Universidade Federal de Mato Grosso do Sul, parecer n⿳⺈ 3.727.518. 


\section{Resultados}

Participaram da pesquisa 52 enfermeiros, com predomínio do sexo feminino. A idade dos profissionais variou entre 27 e 57 anos, com média de 37,63 $( \pm 0,99)$ anos. A maioria atuava na unidade de urgência e emergência, no turno diurno e tinha como maior grau de formação a especialização.

A maioria dos profissionais relatou que não recebeu treinamento acerca do biofilme em feridas. Entre os que receberam, o treinamento foi de até duas horas para a maioria.

Quanto à frequência com que os enfermeiros se deparavam com feridas complexas, a maioria respondeu entre "às vezes" e "frequentemente". A Tabela 1 descreve a caracterização dos participantes.

Tabela 1. Caracterização demográfica e profissional dos participantes, Campo Grande, Mato Grosso do Sul, Brasil, 2021 ( $\mathrm{n}=$ $52)$.

\begin{tabular}{lc}
\hline Variável & $\mathbf{n}(\boldsymbol{\%})$ \\
\hline Idade (27 a 57 anos) & $37,63 \pm 0,99^{1}$ \\
Sexo & $37(71,2)$ \\
Feminino & $15(28,8)$ \\
Masculino & \\
Setor de atuação & $15(28,8)$ \\
Clínico & $16(30,8)$ \\
Cirúrgico & $21(40,4)$ \\
Urgência e emergência & \\
Turno de trabalho & $33(63,5)$ \\
Diurno & $19(36,5)$ \\
Noturno & \\
Pós-graduação & \\
Nenhum & $03(5,8)$ \\
Especialização & $41(78,8)$ \\
Residência & $01(1,9)$ \\
Mestrado & $07(13,5)$ \\
Recebeu treinamento sobre o biofilme & \\
Não & $38(73,1)$ \\
Sim & $14(26,9)$ \\
Duração do treinamento (n=14) & \\
Até 1 hora & $02(14,3)$ \\
Até 2 horas & $09(64,3)$ \\
Até 4 horas & $02(14,3)$ \\
Mais de 4 horas & $01(7,1)$ \\
Frequência com que depara com feridas complexas & \\
Nunca & $03(5,8)$ \\
Frequentemente vezes & $25(48,1)$ \\
Sempre & $18(34,6)$ \\
& $06(11,5)$ \\
\hline
\end{tabular}


A Figura 1 demonstra a caracterização profissional dos participantes. Observa-se que o tempo de atuação no setor variou entre um e 17 anos, com média de atuação de 3,75 $( \pm 0,37)$ anos. Já o tempo de experiência profissional variou entre um e 33 anos, com tempo médio de 11,02 $( \pm 0,88)$ anos. Esses dados foram menores que o tempo de formação na área que variou de cinco a 24 anos com média de $12,80( \pm 0,87)$ anos.

Figura 1. Caracterização profissional dos participantes de acordo com o tempo de formação, experiência profissional e atuação no setor, Campo Grande, Mato Grosso do Sul, Brasil, 2021 ( $\mathrm{n}=52)$.

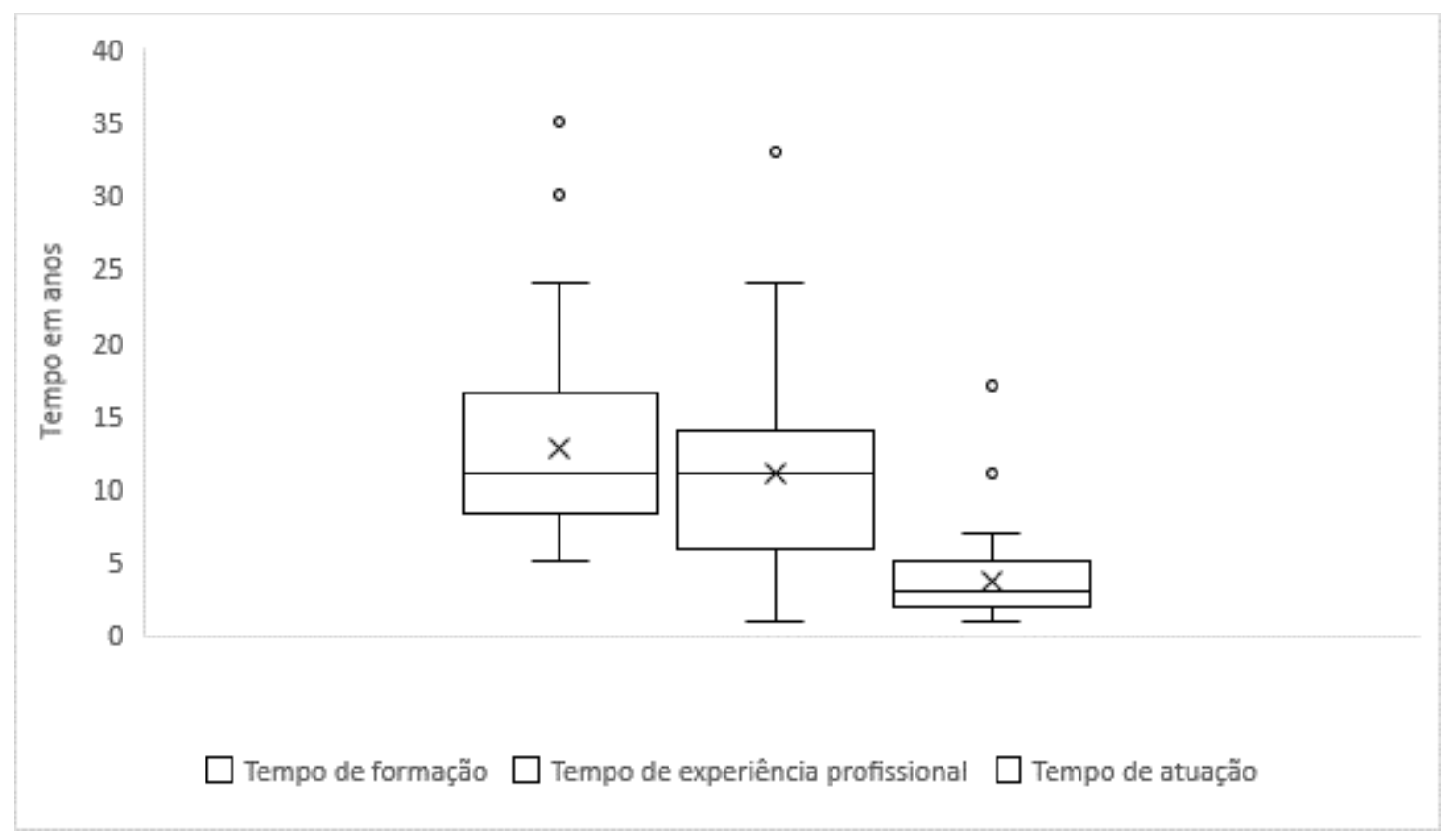

Fonte: Autores.

Na Figura 2 está apresentada a distribuição das respostas dos profissionais em relação ao conhecimento acerca do biofilme em feridas complexas. As perguntas com maior percentual de respostas consideradas corretas ou parcialmente corretas, entre as respostas válidas, foram: "Qual das figuras há biofilme", "Qual o método utilizado para detectar o biofilme em seu local de atuação" e "Qual o tratamento utilizado na prática". A pergunta em que foi observado o maior percentual de respostas incorretas foi "Qual o tratamento ideal para manejar o biofilme". 
Figura 2. Distribuição das respostas sobre o conhecimento dos profissionais acerca do biofilme em feridas complexas, Campo Grande, Mato Grosso do Sul, Brasil, $2021(\mathrm{n}=52)$.

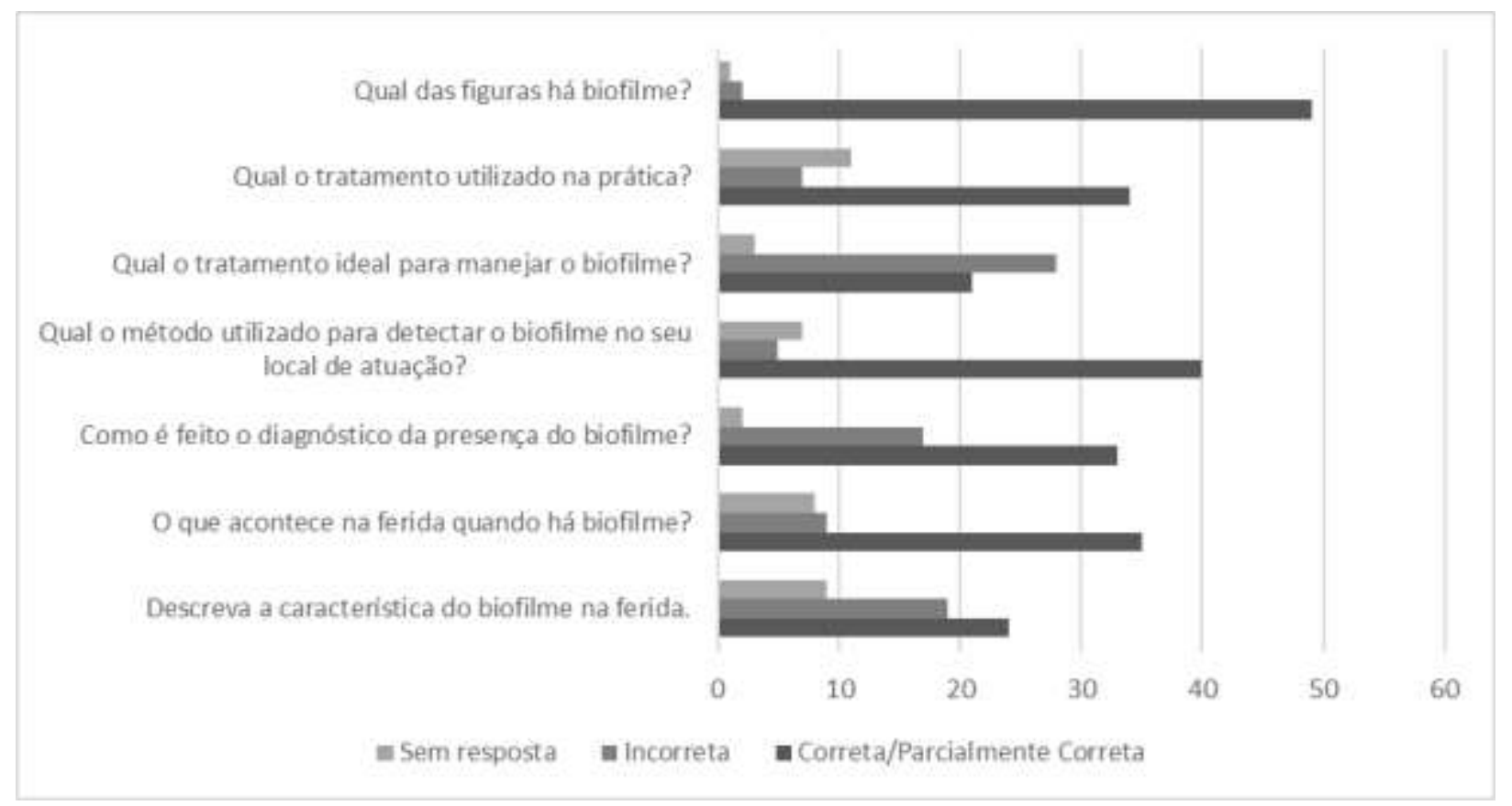

Fonte: Autores.

Quanto a associação entre o sexo e forma de resposta dos participantes, pode-se observar que na pergunta "Descreva a característica do biofilme na ferida", o percentual de mulheres que respondeu de forma correta ou parcialmente correta foi significativamente maior em relação aos homens $(\mathrm{p}=0,005)$. No entanto, não houve diferença significativa no perfil de respostas entre os sexos para as demais perguntas relacionadas e entre as respostas dos enfermeiros em relação à variável idade para nenhuma das perguntas avaliadas.

Também não foi observada associação significativa entre a variável grau de formação para nenhuma das perguntas. Entretanto, os participantes que possuíam alguma especialização ou residência, obtiveram maior percentual de respostas corretas ou parcialmente corretas para a pergunta "Qual das figuras há biofilme" com 40 afirmações (95,2\%).

Referente ao grau de formação, a maioria dos profissionais possuía ao menos uma especialização, das quais 26 em área não assistencial onde as principais foram: enfermagem do trabalho com sete formações, cinco em gestão em saúde, dois em regulação e gestão da clínica, três em auditoria em serviço de saúde e dois em controle de infecção hospitalar.

Entre as áreas de formação assistencial $(n=34)$, predominou a pós-graduação em urgência e emergência (n=12), seguida pela pós-graduação em saúde pública ou saúde da família (n=10) e terapia intensiva (n=05).

Apesar de não haver significância estatística entre as perguntas e a variável tempo de experiência profissional, podese verificar que os enfermeiros que possuíam maior tempo de experiência profissional tiveram maior número de respostas incorretas, quando comparado àqueles que possuíam menor tempo de experiência na área.

O mesmo ocorreu em relação ao setor de atuação e a forma de resposta dos participantes para as perguntas avaliadas. Embora não tenha sido observada associação estatística, profissionais lotados na unidade de urgência e emergência apresentaram maior percentual de respostas incorretas para a pergunta "Qual o tratamento ideal para manejar o biofilme" com 13 afirmativas $(65 \%)$.

A Tabela 3 apresenta os resultados da associação entre o tempo de atuação no setor e a forma de resposta dos enfermeiros. Os participantes que responderam de forma correta ou parcialmente correta a pergunta "Descreva a característica 
do biofilme na ferida" tiveram maior tempo de atuação no setor do que aqueles que responderam de forma incorreta $(\mathrm{p}=0,024)$. Para as demais perguntas não houve diferença estatística.

Tabela 3. Associação entre o conhecimento dos enfermeiros acerca do biofilme em feridas complexas em relação ao tempo de atuação no setor, Campo Grande, Mato Grosso do Sul, Brasil, 2021 ( $\mathrm{n}=52)$.

\begin{tabular}{|c|c|c|c|}
\hline \multirow[b]{2}{*}{ Pergunta } & \multicolumn{3}{|c|}{ Resposta $^{1}$} \\
\hline & $\begin{array}{c}\text { Correta ou } \\
\text { parcialmente } \\
\text { correta }\end{array}$ & Incorreta & Valor de $\mathbf{p}^{2}$ \\
\hline Descreva a característica do biofilme na ferida. & $4,04 \pm 0,44$ & $2,68 \pm 0,33$ & $\mathbf{0 , 0 2 4}$ \\
\hline O que acontece na ferida quando há biofilme? & $3,43 \pm 0,34$ & $3,33 \pm 0,60$ & 0,899 \\
\hline Como é feito o diagnóstico da presença do biofilme? & $3,55 \pm 0,37$ & $4,00 \pm 0,87$ & 0,576 \\
\hline Qual o método utilizado para detectar o biofilme no seu local de atuação? & $3,78 \pm 0,46$ & $3,20 \pm 0,66$ & 0,671 \\
\hline Qual o tratamento ideal para manejar o biofilme? & $3,33 \pm 0,49$ & $3,96 \pm 0,57$ & 0,426 \\
\hline Qual o tratamento utilizado na prática? & $3,41 \pm 0,36$ & $4,86 \pm 2,12$ & 0,239 \\
\hline Qual das figuras há biofilme? & $3,67 \pm 0,39$ & $5,00 \pm 0,00$ & 0,495 \\
\hline
\end{tabular}

Nota $^{1}$ : os resultados estão apresentados em média \pm erro padrão da média; ${ }^{2}$ valor de p no teste t-student. Fonte: Autores.

Os resultados da associação entre o turno de trabalho dos participantes e a forma de resposta (correta ou parcialmente correta e incorreta) estão apresentados na Tabela 4. Em relação à pergunta “Qual o tratamento ideal para manejar o biofilme", o percentual de enfermeiros que trabalhavam no turno diurno que responderam de forma correta ou parcialmente correta foi significativamente maior quando comparado àqueles que trabalhavam no turno noturno ( $\mathrm{p}=0,015)$. No entanto, não houve diferença significativa no perfil de respostas entre enfermeiros que trabalhavam em turnos diferentes para as demais perguntas relacionadas. 
Tabela 4. Associação entre o turno de trabalho dos enfermeiros e o conhecimento sobre biofilme em feridas complexas, Campo Grande, Mato Grosso do Sul, Brasil, $2021(\mathrm{n}=52)$.

\begin{tabular}{|c|c|c|c|}
\hline \multirow[b]{2}{*}{ Pergunta } & \multicolumn{2}{|c|}{ Turno de trabalho } & \multirow[b]{2}{*}{ Valor de $\mathbf{p}$} \\
\hline & $\begin{array}{c}\text { Diurno } \\
\text { n }(\%)\end{array}$ & $\begin{array}{c}\text { Noturno } \\
\text { n (\%) }\end{array}$ & \\
\hline \multicolumn{4}{|l|}{ Descreva a característica do biofilme na ferida. } \\
\hline Correta ou parcialmente correta & $14(50,0)$ & $10(66,7)$ & \multirow{2}{*}{0,349} \\
\hline Incorreta & $14(50,0)$ & $05(33,3)$ & \\
\hline \multicolumn{4}{|l|}{ O que acontece na ferida quando há biofilme? } \\
\hline Correta ou parcialmente correta & $25(82,8)$ & $11(73,3)$ & \multirow[t]{2}{*}{0,464} \\
\hline Incorreta & $05(17,2)$ & $04(26,7)$ & \\
\hline \multicolumn{4}{|l|}{ Como é feito o diagnóstico da presença do biofilme? } \\
\hline Correta ou parcialmente correta & $22(66,7)$ & $11(64,7)$ & \multirow{2}{*}{1,000} \\
\hline Incorreta & $11(33,3)$ & $06(35,3)$ & \\
\hline \multicolumn{4}{|c|}{ Qual o método utilizado para detectar o biofilme no seu local de atuação? } \\
\hline Correta ou parcialmente correta & $27(87,1)$ & $13(92,9)$ & \multirow{2}{*}{1,000} \\
\hline Incorreta & $04(12,9)$ & $01(7,1)$ & \\
\hline \multicolumn{4}{|l|}{ Qual o tratamento ideal para manejar o biofilme? } \\
\hline Correta ou parcialmente correta & $18(56,3)$ & $03(17,6)$ & \multirow{2}{*}{0,015} \\
\hline Incorreta & $14(43,8)$ & $14(82,4)$ & \\
\hline \multicolumn{4}{|l|}{ Qual o tratamento utilizado na prática? } \\
\hline Correta ou parcialmente correta & $25(86,2)$ & $09(75,0)$ & \multirow{2}{*}{0,398} \\
\hline Incorreta & $04(13,8)$ & $03(25,0)$ & \\
\hline \multicolumn{4}{|l|}{ Qual das figuras há biofilme? } \\
\hline Correta ou parcialmente correta & $33(100,0)$ & $16(88,9)$ & \multirow{2}{*}{0,120} \\
\hline Incorreta & $00(0,0)$ & $02(11,1)$ & \\
\hline
\end{tabular}

Nota $^{1}$ : valor de p no teste exato de Fisher. Fonte: Autores.

Mais de $50 \%$ dos enfermeiros $(\mathrm{n}=28)$ responderam de forma incorreta a pergunta "Qual o tratamento ideal para manejar o biofilme". Entre as respostas corretas, o uso de antissépticos, a troca de curativo diária, o polihexametileno biguanida (PHMB) e a terapia antimicrobiana tópica e sistêmica foram as opções terapêuticas mais citadas pelos profissionais.

Quanto ao tratamento utilizado na prática, a maioria dos profissionais $(n=34 ; 65,4 \%)$ respondeu de forma parcialmente correta e nenhum dos enfermeiros respondeu de forma correta. A solução de PHMB e a terapia com antissépticos foram citados como os itens mais utilizados na prática clínica dos enfermeiros.

Embora não tenha sido observada significância estatística entre ter ou não recebido treinamento acerca do biofilme e a forma de resposta dos participantes, aqueles que não receberam, obtiveram maior percentual de erros com 15 afirmativas incorretas $(41,7 \%)$ para a pergunta "Como é feito o diagnóstico da presença do biofilme” quando comparado aos profissionais que receberam o treinamento.

Na pergunta "Descreva a característica do biofilme na ferida", o percentual de enfermeiros que "nunca" ou "às vezes" se deparavam com feridas complexas e que respondeu de forma correta ou parcialmente correta, foi significativamente maior que os enfermeiros que "frequentemente" ou "sempre" se deparavam com a lesão ( $\mathrm{p}=0,033)$. No entanto, não houve diferença significativa para as demais perguntas relacionadas, conforme demonstrado na tabela abaixo. 
Tabela 5: Associação entre a frequência com que os enfermeiros se deparavam com as feridas complexas e a forma de resposta sobre o conhecimento acerca do biofilme em feridas complexas, Campo Grande, Mato Grosso do Sul, Brasil, 2021, $(n=52)$.

\begin{tabular}{|c|c|c|c|}
\hline \multirow{2}{*}{ Pergunta } & \multicolumn{2}{|c|}{$\begin{array}{l}\text { Frequência com que depara com esse } \\
\text { tipo de ferida }\end{array}$} & \multirow{2}{*}{ Valor de $\mathbf{p}^{2}$} \\
\hline & $\begin{array}{c}\text { Nunca/ } \\
\text { Às vezes }\end{array}$ & $\begin{array}{l}\text { Frequentemente } \\
\text { Sempre }^{1}\end{array}$ & \\
\hline \multicolumn{4}{|c|}{ Descreva a característica do biofilme na ferida. } \\
\hline Correta ou parcialmente correta & $16(72,7) \mathrm{a}$ & $8(38,1) b$ & \multirow{2}{*}{$\mathbf{0 , 0 3 3}$} \\
\hline Incorreta & $6(27,3) b$ & $13(61,9) \mathrm{a}$ & \\
\hline \multicolumn{4}{|c|}{ O que acontece na ferida quando há biofilme? } \\
\hline Correta ou parcialmente correta & $20(87,0)$ & $15(71,4)$ & \multirow{2}{*}{0,272} \\
\hline Incorreta & $3(13,0)$ & $6(28,6)$ & \\
\hline \multicolumn{4}{|c|}{ Como é feito o diagnóstico da presença do biofilme? } \\
\hline Correta ou parcialmente correta & $17(63,0)$ & $16(69,6)$ & \multirow{2}{*}{0,767} \\
\hline Incorreta & $10(37,0)$ & $7(30,4)$ & \\
\hline \multicolumn{4}{|c|}{$\begin{array}{l}\text { Qual o método utilizado para detectar o biofilme em seu local de } \\
\text { atuação? }\end{array}$} \\
\hline Correta ou parcialmente correta & $21(91,3)$ & $19(86,4)$ & \multirow{2}{*}{0,665} \\
\hline Incorreta & $2(8,7)$ & $3(13,6)$ & \\
\hline \multicolumn{4}{|c|}{ Qual o tratamento ideal para manejar o biofilme? } \\
\hline Correta ou parcialmente correta & $10(37,0)$ & $11(50,0)$ & \multirow{2}{*}{0,398} \\
\hline Incorreta & $17(63,0)$ & $11(50,0)$ & \\
\hline \multicolumn{4}{|l|}{ Qual o tratamento utilizado na prática? } \\
\hline Correta ou parcialmente correta & $17(85,0)$ & $17(81,0)$ & \multirow{2}{*}{1,000} \\
\hline Incorreta & $3(15,0)$ & $4(19,0)$ & \\
\hline \multicolumn{4}{|l|}{ Qual das figuras há biofilme? } \\
\hline Correta ou parcialmente correta & $26(92,9)$ & $23(100,0)$ & \multirow{2}{*}{0,495} \\
\hline Incorreta & $2(7,1)$ & $0(0,0)$ & \\
\hline
\end{tabular}

Nota $^{1}$ : os resultados estão apresentados em frequência absoluta (frequência relativa). ${ }^{2}$ Valor de p no teste exato de Fisher. Fonte: Autores.

\section{Discussão}

Este estudo buscou analisar o conhecimento de enfermeiros acerca do biofilme em feridas complexas, complicação comum na rotina de trabalho destes profissionais. Participaram da pesquisa 52 enfermeiros, com predomínio do sexo feminino, dado que vem ao encontro de outros estudos que observaram maior proporção de mulheres entre os pesquisados (Faria, et al., 2016; Rabeh, Palfreyman, Souza, Bernardes, \& Caliri, 2017).

A média de idade dos participantes foi de 37,63 anos, resultados semelhantes foram encontrados em outro estudo onde o tempo de formação acadêmica variou entre cinco e 24 anos, com média de 12,80 anos (Agra, et al., 2017). Uma pesquisa realizada com 316 profissionais de saúde observou que o tempo de formação dos enfermeiros variou de cinco a 10 anos, média de 8,3 anos (Malta, Araújo, Brito, \& Pinho, 2020). Quanto ao tempo de atuação no setor, a média foi de 3,75 anos, o que corrobora os achados na literatura (Santos, Camelo, Laus, \& Leal, 2015). 
Foi observado que as mulheres obtiveram maior número de acertos ao descreverem as características do biofilme na ferida, dados que podem estar relacionados ao predomínio do sexo feminino na amostra que reflete a atuação marcante da população feminina na área da enfermagem (Souza, Loureiro, \& Batiston, 2020).

Embora a maioria dos participantes tenha descrito corretamente as características do biofilme na ferida, foi evidenciado maior índice de respostas incorretas quando relacionadas ao tratamento ideal para manejar o biofilme, o que sugere que os enfermeiros desconhecem o tratamento correto para esse tipo de lesão. Afirmativa também observada em uma pesquisa que avaliou o nível de conhecimento de 18 enfermeiros que atuavam no tratamento de feridas e que identificou baixo nível de conhecimento referente ao tema biofilme (Colares, Luciano, Neves, Tipple, \& Júnior, 2019).

Além disso, foi possível observar que a maioria dos enfermeiros não soube categorizar o PHMB de acordo com sua ação na ferida, um potente antisséptico para ser utilizado no tratamento contra o biofilme (Zheng, Wang, \& Ma, 2021).

Quanto ao tratamento utilizado na prática, percebe-se que nenhum profissional respondeu corretamente, o que reforça o desconhecimento teórico e por consequência, prático, acerca do manejo do biofilme, que consiste na avaliação prévia da lesão, utilização de terapia antisséptica e a antibioticoterapia tópica e sistêmica e quando da presença de tecido desvitalizado com sinais de infecção, sugere-se a associação do desbridamento (Percival, et al., 2015; Wei, et al., 2019; Oliveira, et al., 2021).

Vale ressaltar que o tratamento é considerado um desafio devido a característica polimicrobiana dos microorganismos, nos quais exige adequado conhecimento por parte dos profissionais de saúde, especialmente dos enfermeiros, pois são esses os profissionais que frequentemente realizam a avaliação das feridas bem como o seu tratamento (Cruz, Acioly, Nóbrega, \& Oliveira, 2016).

Não houve associação estatística entre o conhecimento dos profissionais acerca do biofilme em feridas complexas e a idade, pós-graduação, tempo de experiência profissional, setor de atuação e realização de treinamentos acerca da temática. Achados semelhantes foram encontrados na literatura em que pesquisadores também não encontraram significância estatística entre o escore geral de acertos e a idade, tempo de experiência e se possui ou não pós-graduação (Paula, Souza, Almeida, \& Santos, 2019).

Entretanto, em relação ao tempo de experiência profissional, percebe-se que os enfermeiros que possuíam maior tempo de experiência responderam de forma incorreta quatro das sete questões relativas à temática. Pesquisadores também identificaram baixo conhecimento acerca do manejo com feridas em profissionais mais experientes (Ferreira, et al., 2014). Dado que permite inferir que o tempo de experiência pode ser um fator determinante para a atualização profissional e que quanto maior a experiência na área, maior a acomodação profissional em sua rotina de trabalho, o que pode acarretar um menor interesse na busca por novos conhecimentos.

De modo a divergir desta afirmativa, um estudo realizado com 22 enfermeiros que atuavam na área de oncologia demonstrou que os profissionais menos experientes apresentaram déficit no conhecimento e prática no que diz respeito ao manejo de feridas tumorais malignas, o que também pode ser associado a uma menor busca por atualização (Agra, et al., 2017).

Ao contrário da experiência profissional, participantes que possuíam alguma especialização ou residência, foram mais assertivos ao identificar clinicamente o biofilme nas imagens. Um estudo que realizou a adaptação cultural do instrumento Pressure Ulcer Knowledge Test (PZ-PUKT) verificou que enfermeiros que possuíam especialização clínica apresentaram maior percentual de acertos do que os demais participantes do estudo (Rabeh, et al., 2017). Esses achados sugerem que ter alguma especialização na área da enfermagem pode contribuir para o aprimoramento do raciocínio clínico do enfermeiro.

Quanto ao setor de atuação, percebe-se que a maioria dos profissionais que atuavam no setor de urgência não soube descrever o tratamento ideal para manejar o biofilme. Este resultado pode ser justificado pelo fato de se tratar de um ambiente 
que atende frequentemente pacientes com risco iminente de vida, superlotação, demandas administrativas e assistenciais, o que resulta em sobrecarga de trabalho aos enfermeiros e com isso prejudica a avaliação clínica das lesões (Rabelo, et al., 2020).

A maioria dos participantes assinalou que não havia recebido treinamento acerca do biofilme. Um estudo realizado com 197 profissionais de enfermagem identificou que 54,8\% dos enfermeiros não havia recebido capacitação específica sobre prevenção e tratamento de lesão por pressão (Souza, et al., 2020). Estes dados sugerem que a ausência de atividades de ensinoaprendizagem possa ter contribuído para o percentual significativo de erros nas questões referentes ao diagnóstico e tratamento do biofilme.

Outro estudo (Macedo, Freitas, Dionísio, \& Torres, 2019) identificou que 88\% dos participantes também não havia recebido treinamento sobre o cuidado com feridas, além disso a questão "Escolha de cobertura" foi a que apresentou maior número de respostas incorretas, o que demonstra a necessidade de constante atualização por parte dos enfermeiros para melhor manejo e tratamento desse tipo de lesão.

Por fim, embora este estudo seja o primeiro a avaliar o conhecimento de enfermeiros acerca do biofilme no estado do Mato Grosso do Sul apresenta algumas limitações, tais como o tamanho da amostra e inclusão de apenas uma instituição. Além disso, a escassez de estudos na literatura, bem como a falta de instrumentos validados para avaliar o conhecimento de enfermeiros acerca do biofilme em feridas complexas dificultou o processo de análise e comparação entre os dados.

\section{Conclusão}

Foi possível identificar algumas lacunas no conhecimento dos profissionais enfermeiros frente ao biofilme em feridas complexas, principalmente no que diz respeito ao diagnóstico e tratamento. Entre os fatores que podem ter contribuído para estes resultados, destacamos o déficit de atividades voltadas ao ensino-aprendizagem na instituição, especificamente no que diz respeito aos cuidados com as feridas complexas e a falta de protocolos clínicos que poderiam ajudar os enfermeiros na sistematização da assistência.

\section{Referências}

Agra, G., Medeiros, M. V. S., Brito, D. T. F., Souza, A. T. O., Formiga, N. S., \& Costa, M. M. L. (2017). Conhecimento e prática de enfermeiros no cuidado a pacientes com feridas tumorais malignas. Revista Cuidarte, 8(3): 1849-62. doi: http://dx.doi.org/10.15649/cuidarte.v8i3.441.

Colares, C. M. P., Luciano, C. C., Neves, H. C. C., Tipple, A. F. V., \& Júnior, H. G. (2019). Cicatrização e tratamento de feridas: a interface do conhecimento à prática do enfermeiro. Enfermagem em Foco, 10(3): 52-58. doi: https://doi.org/10.21675/2357-707X.2019.v10.n3.2232.

Cruz, R. A. O., Acioly, C. M. C., Nóbrega, V. K. M., \& Oliveira, P. S. (2016). Feridas complexas e o biofilme: atualização de saberes e práticas para enfermagem. Revista Rede de Cuidado em Saúde, 10(3): ISSN-19826451. Recuperado de http://publicacoes.unigranrio.edu.br/index.php/rcs/article/view/3084/2093.

Duim, E., Sá, F. H. C., Duarte, Y. A. O., Oliveira, R. C. B., \& Lebrão, M. L. (2015). Prevalência e características das feridas em pessoas idosas residentes na comunidade. Escola de Enfermagem da USP, 49(Esp):51-57. doi: https://doi.org/10.1590/S0080-623420150000700008.

Faria, G. B. G., Prado, T. N., Lima, E. F. A., Rogenski, N. M. B., Borghardt, A. T., \& Massaroni, L. (2016). Conhecimento e prática dos enfermeiros sobre o cuidado com feridas. Revista de Enfermagem. UFPE On Line, 10(12): 4532-8. doi: 10.5205/reuol.9978-88449-6-ED1012201614.

Ferreira, A. M. F., Rigotti, M. A., Barcelos, L. S., Simão, C. M. F. S., Ferreira, D. N., \& Gonçalves, R. Q. (2014). Conhecimento e prática de enfermeiros sobre cuidados aos pacientes com feridas. Revista de Pesquisa: Cuidado é Fundamental Online, 6(3): 1178-1190. doi: 10.9789/2175-5361.2014v6n3p1178.

Ferro, T. A. F., Souza, E. B., Suarez, M. A. M., Rodrigues, J. F. S., Pereira, D. M. S., Mendes, S. J. F., Gonzaga, L. F., Machado, M. C. A. M., Bomfim, M. R. Q., Calixto, J. B., Arbiser, J. L., Monteiro-Neto, V., André, E., \& Fernandes, E. S. (2019). Topical application of cinnamaldehyde promotes faster healing of skin wounds infected with pseudomonas aeruginosa. Molecules, 24(8): 1627. Retrieved from https://pubmed.ncbi.nlm.nih.gov/31027179/.

Franco, V. Q., Souza, N. V. D. O., Pires, A. S., Rafael, R. M. R., Nascimento, D. C., \& Nunes, M. L. G. (2018). Tecnologia da espuma de poliuretano com prata iônica e sulfadiazina de prata: aplicabilidade em feridas cirúrgicas infectadas. Revista de Pesquisa: Cuidado é Fundamental Online, 10(2): 441-449. doi: http://dx.doi.org/10.9789/2175-5361.2018.v10i2.441-449.

Jara, C. P., Silva, J. L. G., Zanchetta, F. C., Rojo, T., \& Lima, M. H. M. (2017). Biofilme e feridas crônicas: reflexões para o cuidado de enfermagem. Enfermagem atual, 81(19). Recuperado de https://revistaenfermagematual.com.br/index.php/revista/article/view/324. 
Research, Society and Development, v. 10, n. 13, e232101321223, 2021

(CC BY 4.0) | ISSN 2525-3409 | DOI: http://dx.doi.org/10.33448/rsd-v10i13.21223

Macedo, E. A. B., Freitas, C. C. S., Dionisio, A. J., \& Torres, G. V. (2019). Knowledge of the care of wounded patients: evidence of validity of an instrument. Revista Brasileira de Enfermagem, 72(6): 1562-70. doi: https://doi.org/10.1590/0034-7167-2018-0643.

Malta, E. M. B. R., Araújo, D. D., Brito, M. F. S. F., \& Pinho, L. (2020). Práticas de profissionais da Atenção Primária à Saúde (APS) no cuidado a idosos com demência. Interface, 24(Supl. 1): e190449. doi: https://doi.org/10.1590/Interface.190449.

Murphy, C., Atkin, L., Swanson, T., Tachi, M., Tan, Y. K., Ceniga, M. V., Weir, D., Wolcott, R., Cernohorská, J., Ciprandi, G., Dissemond, J., James, G. A., Hurlow, J., Martinez, J. L. L., Mrozikiewicz-Rakowska, B., \& Wilson, P. (2020). Defying hard-to-heal wounds with an early antibiofilm intervention strategy: wound hygiene. Journal of Wound Care, 29(Sup3b). doi: https://doi.org/10.12968/jowc.2020.29.Sup3b.S1.

Mihai, M. M., Preda, M., Lungu, I., Gestal, M. C., Popa, M. I., \& Holban, A. M. (2018). Nanocoatings for Chronic Wound Repair-Modulation of Microbial Colonization and Biofilm Formation. International Journal of Molecular Sciences, 19(4): 1179. doi: https://doi.org/10.3390/ijms19041179.

Oliveira, F. T., Lima, G. K. S., Santos, I. M. R., Silva, D. P., Bernardo, T. H. L., Bastos, M. L. A., Silva, P. S. G., \& Barros, D. A. (2021). Microrganismos e Resistência Antimicrobiana em feridas complexas. Research, Society and Development, 10(2): e10110212161, 2021. doi: http://dx.doi.org/10.33448/rsdv10i2.12161.

Paula, V. A. A., Souza, I. D., Almeida, R. L. M., \& Santos, K. B. (2019). O conhecimento dos enfermeiros assistenciais no tratamento de feridas. HU Revistas, 45(3): 295-303. doi: 10.34019/1982-8047.2019.v45.28666.

Percival, S. L., Vuotto, C., Donelli, G., \& Lipsky, B. A. (2015). Biofilms and wounds: an identification algorithm and potential treatment options. Advances in Wound Care (New Rochelle), 4(7): 389-397. doi: https://doi.org/10.1089/wound.2014.0574.

Rabeh, S. A. N., Palfreyman, S., Souza, C. B. L., Bernardes, R. M., \& Caliri, M. H. L. (2017). Cultural adaptation of the Pieper-Zulkowski Pressure Ulcer Knowledge Test for use in Brazil. Revista Brasileira de Enfermagem, 71(4): 1977-84. doi: https://doi.org/10.1590/0034-7167-2017-0029.

Rabelo, S. K., Lima, S. B. S., Santos, J. L. G., Costa, V. Z., Reisdorfer, E., Santos, T. M., \& Gracioli, J. C. (2020). Nurses work process in an emergency hospital service. Revista Brasileira de Enfermagem, 73(5): e20180923. doi:10.1590/0034-7167-2018-0923.

Santos, F. C., Camelo, S. H. H., Laus, A. M., \& Leal, L. A. (2015). El enfermero de unidades hospitalarias oncológicas: perfil y capacitación profesional. Enfermaría Global, 14(38): 301-312. Recuperado de http://scielo.isciii.es/scielo.php?script=sci_arttext\&pid=S1695-61412015000200016.

Souza, M. C., Loureiro, M. D. R., \& Batiston, A. P. (2020) Organizational culture: prevention, treatment, and risk management of pressure injury. Revista Brasileira de Enfermagem, 73(3): e20180510. doi: https://doi.org/10.1590/0034-7167-2018-0510.

Wei, D., Zhu, X. M., Chen, Y. Y., Li, X. Y., Chen YP., Liu HY., \& Zhang, M. (2019). Chronic wound biofilms diagnosis and therapeutic strategies. Chinese Medical Journal, 132(22): 2737-2744. doi: 10.1097/CM9. 0000000000000523.

Zheng, Y., Wang, D., \& Ma, L. Z. (2021). Effect of polyhexamethylene biguanide in combination with undecylenamidopropyl betaine or pslg on biofilm clearance. Int. J. Mol, 22(2), 768. doi: https://doi.org/10.3390/ijms22020768. 